\title{
Establishment and Verification of UPLC-MS/MS Technique for Pharmacokinetic Drug-Drug Interactions of Selinexor with Posaconazole in Rats
}

This article was published in the following Dove Press journal:

Drug Design, Development and Therapy

\author{
Chen-jian Zhou' \\ Hui-jun Wang ${ }^{2}$ \\ Chun-yan Zhou ${ }^{2}$ \\ Chao-fan $\mathrm{Li}^{2}$ \\ Ming-Jia Zhu ${ }^{2}$ \\ Xiang-jun $\mathrm{Qiu}^{2}$ \\ 'Department of Pharmacy, Wenzhou \\ Central Hospital, Wenzhou, 325027, \\ People's Republic of China; ${ }^{2}$ School of \\ Basic Medical Sciences, Henan University \\ of Science and Technology, Luoyang, \\ 47I023, People's Republic of China
}

Correspondence: Xiang-jun Qiu School of Basic Medicine, Henan University of Science and Technology, 263

Kaiyuan Avenue, Luoyang, 47I023,

People's Republic of China

Tel +8613698882699

Fax +86379-64830346

Email lyxiangjun@I26.com
Background: A method for the determination of selinexor by UPLC-MS/MS was established to study the effect of posaconazole on the pharmacokinetics of selinexor in rats.

Methods: The experiment rats were divided into group A $(0.5 \% \mathrm{CMC}-\mathrm{Na})$ and group $\mathrm{B}$ (posaconazole, $20 \mathrm{mg} / \mathrm{kg}$ ), 6 rats in each group. 30 minutes after administration of $0.5 \%$ CMC-Na or posaconazole, all the rats were given selinexor $(8 \mathrm{mg} / \mathrm{kg})$, and plasma samples were collected. The plasma samples underwent acetonitrile protein precipitation, and were separated by UPLC on an Acquity UPLC BEH C18 column with gradient elution. Acetonitrile and $0.1 \%$ formic acid were used as the mobile phases. The analyte detection was used a Xevo TQ-S triple quadrupole tandem mass spectrometer and multiple reaction monitoring (MRM) for analyte monitoring. We use acetonitrile for protein precipitation.

Results: Selinexor had good linearity (1.0-1000 ng/mL, $\left.\mathrm{r}^{2}=0.9962\right)$, and the accuracy and precision, recovery rate and matrix effects(ME) were also met the FDA approval guidelines. Compared with group $\mathrm{A}$, the $\mathrm{C}_{\max }, \mathrm{AUC}_{(0-\mathrm{t})}$ and $\mathrm{AUC}_{(0-\infty)}$ of selinexor in group $\mathrm{B}$ increased by $60.33 \%, 48.28 \%$ and $48.27 \%$, and $\mathrm{T}_{\max }$ increased by $53.92 \%$, CLz/F reduced by $32.08 \%$. Conclusion: This bioanalysis method had been applied to the study of drug interactions in rats. It was found that posaconazole significantly increased the concentration of selinexor in rats. Therefore, when selinexor and posaconazole are combined, we should pay attention to the possible drug-drug interactions to reduce adverse reactions.

Keywords: UPLC-MS/MS, selinexor, posaconazole, pharmacokinetics, DDIs, rats

\section{Introduction}

Selinexor (KPT-330, Figure 1A) is the world's first, oral, small molecule selective inhibition of nuclear export (SINE). ${ }^{1}$ Exportin-1 (XPO1) is a tumor suppressor protein, it can act on transcription factors, and growth regulators and oncoprotein mRNA. ${ }^{2-4}$ The overexpression of XPO-1 leads to an increase in the output of tumor suppressor and regulatory proteins, which can lead to enhanced signal transduction for abnormal cell growth and prevent apoptosis. ${ }^{5}$ XPO1 is overexpressed in most cancer types and is associate with poor prognosis of acute myeloid leukemia, glioma, pancreatic cancer, cervical cancer and ovarian cancer. ${ }^{6-10}$ Inhibition of XPO-1 can force tumor suppressor proteins and growth regulators to remain in the nucleus, leading to cancer cell apoptosis. ${ }^{5}$

Selinexor works by binding and inhibiting nuclear export protein XPO1. ${ }^{11}$ It causing tumor suppressor protein to accumulate in the nucleus, thereby restarting 


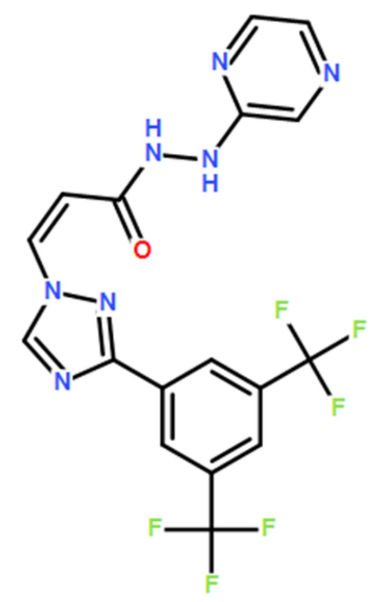

A

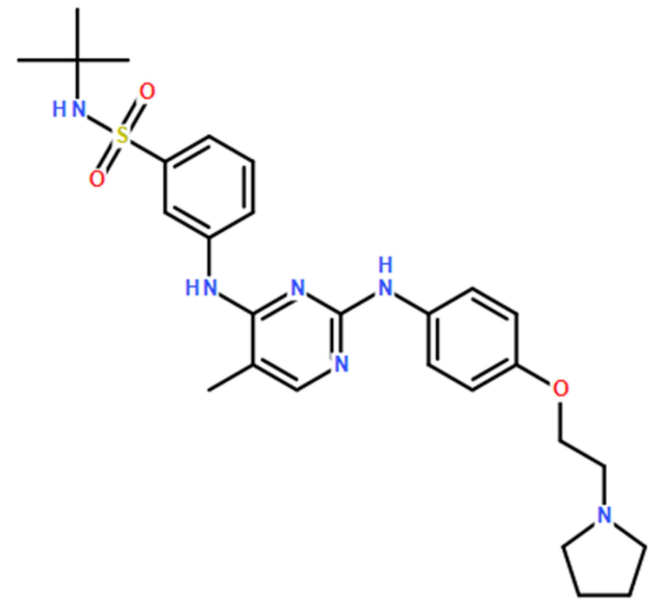

B

Figure I The chemical structure of selinexor (A) and fedratinib (IS, (B)).

and amplifying its function as a tumor suppressor, leading to apoptosis of cancer cells, while largely protecting normal cells from damage..$^{2-4}$

On July 3, 2019, the US FDA approved Selinexor in combination with low-dose dexamethasone for the treatment of relapsed or refractory multiple myeloma (RRMM). ${ }^{1,12}$ Jan 29, 2021, Karyopharm receives positive CHMP opinion for $\mathrm{NEXPOVIO}^{\circledR}$ (selinexor) for the treatment of patients with refractory multiple myeloma. ${ }^{13}$ In clinic, the recommended starting dose of Selinexor is $80 \mathrm{mg}$, and the effect of food on its pharmacokinetics is not significant. ${ }^{1,2}$ In cancer patients, $\mathrm{t}_{\max }$ is about $2-4 \mathrm{~h}$; plasma protein binding rate is about $95 \%$; $\mathrm{t}_{1 / 2}$ is $6-8 \mathrm{~h} ; \mathrm{CLz} / \mathrm{F}$ is $17.9 \mathrm{~L} / \mathrm{h}$. Selinexor is mainly metabolized by CYP3A4. ${ }^{1}$

The most common adverse reactions of Selinexor $(\geq 20 \%)$ were thrombocytopenia, fatigue, nausea, anemia, decreased appetite, decreased weight, diarrhea, vomiting, hyponatremia, neutropenia, leukopenia, constipation, dyspnea and upper respiratory tract infection. In the STORM trial, fatal adverse reactions occurred in $9 \%$ of patients. Serious adverse reactions occurred in $58 \%$ of patients. Treatment discontinuation rate due to adverse reactions was $27 \%{ }^{13}$

Drug-drug interactions (DDIs) often occur, especially for patients with multiple underlying diseases who use various kinds of drugs. In clinical practice, polypharmacy is a common problem and results in the increased risks of DDIs. ${ }^{14}$ Due to fungal infection in patients with multiple myeloma, antifungal drugs are often used in clinic. Therefore, selinexor and posaconazole may be used at the same time in clinic. Because selinexor and posaconazole were both metabolized by the CYP3A4 enzyme, so when the two drugs were used at the same time, the exposure of selinexor would increase due to the inhibition of CYP3A4 enzyme by posaconazole. Thereby enhancing drug concentrations and DDIs.

UPLC-MS/MS had been widely used for the determination of drug concentrations in various biological matrices, which had many advantages such as short time, high sensitivity and strong specificity. ${ }^{15,16}$ This paper presented a new, rapid and accurate UPLC-MS/MS method for determining the concentration of selinexor and investigated the effects of posaconazole on the pharmacokinetics of selinexor using fedratinib as the internal standard (IS, Figure 1B).

\section{Methods}

\section{Chemicals Materials}

Selinexor and posaconazole (purity over 98\%) was provided by Jiangsu Aikang Biomedicine R \& D Co., Ltd, and fedratinib (purity over 98\%, IS) was obtained from Beijing sunflower and technology development CO., LTD (Beijing, China). Acetonitrile and formic acid were analytical grade, and water was deionized water.

\section{Chromatographic Conditions}

The Waters ACQUITY UPLC I-Class (Milford, MA, USA) used in this experiment and the Acquity BEH C18 column $(2.1 \mathrm{~mm} \times 100 \mathrm{~mm}, 1.7 \mu \mathrm{m})$ were used as the separation column. The mobile phase used $0.1 \%$ formic acid (A) and acetonitrile (B), and the gradient elution is shown in Table 1. 
Table I The Gradient of Mobile Phase

\begin{tabular}{|l|c|c|c|c|c|}
\hline $\begin{array}{c}\text { Time } \\
\text { (Min) }\end{array}$ & $\begin{array}{c}\text { A } \\
\text { (\%) }\end{array}$ & $\begin{array}{c}\text { B } \\
\text { (\%) }\end{array}$ & $\begin{array}{c}\text { Flow Rate } \\
(\mathbf{m L} / \mathbf{m i n})\end{array}$ & $\begin{array}{c}\text { Temperature } \\
\left({ }^{\circ} \mathbf{C}\right)\end{array}$ & Curve \\
\hline 0 & 85 & 15 & 0.35 & 45 & 0 \\
1.00 & 85 & 15 & 0.35 & 45 & 6 \\
1.10 & 15 & 85 & 0.35 & 45 & 6 \\
2.10 & 15 & 85 & 0.35 & 45 & 6 \\
2.20 & 85 & 15 & 0.35 & 45 & 6 \\
3.00 & 85 & 15 & 0.35 & 45 & 6 \\
\hline
\end{tabular}

\section{MS Conditions}

Mass spectrometry analysis was performed on a Waters Xevo TQ-S triple quadrupole mass spectrometer. Multiple reaction monitoring (MRM) conditions are shown in Table 2.

\section{Animal Experiments}

The Laboratory Animal Center of Henan University of Science and Technology (Luoyang, China) provides male Sprague-Dawley rats $(200 \pm 20 \mathrm{~g})$, and the animal certification was SCXK (Hubei) 2007-0001. Necessary approval from the Animal Ethics Committee of the animal laboratory of Henan university of science and technology was obtained to carry out the experiments, and the experimental content was approved according to the Laboratory animals-guidelines for ethical review of welfare ( $\mathrm{GB} / \mathrm{T}$ 35892-2018). The institutional approval number for the preclinical study of this experiment was 2019100003. All authors have read the NC3Rs ARRIVE guidelines.

Twelve rats were randomly divided into two groups and administered orally according to body weight. Group A was orally given with $0.5 \% \mathrm{CMC}-\mathrm{Na}$ and group B was orally given with $20 \mathrm{mg} / \mathrm{kg}$ posaconazole. Thirty minutes later, $8 \mathrm{mg} / \mathrm{kg}$ selinexor was orally administered to each rat. At the time point of $0.33,0.67,1,1.5,2,3,4,6,9,12$, 24 and 48 hours, approximately $0.2 \mathrm{~mL}$ of blood sample was collected from the tail vein of each rat. The blood samples were centrifuged at $10000 \mathrm{rmp} * 10 \mathrm{~min}, 50 \mu \mathrm{L}$ plasma was obtained and stored at $-20^{\circ} \mathrm{C}$ until analysis. All rats were sacrificed by $\mathrm{CO}_{2}$ inhalation.

\section{Solutions Ready}

$10.0 \mathrm{mg}$ of Selinexor and IS standard product were weighed out and dissolved in methanol to prepare the stock solution with a concentration of $1.00 \mathrm{mg} / \mathrm{mL}$. Then, the standard stock solution was gradient diluted to obtain the calibration curve and the working solution of the quality control (QC) samples. The final concentrations obtained by mixing blank plasma with the corresponding standard working solutions were: $1,5,10,50,100,200$, $500,1000 \mathrm{ng} / \mathrm{mL}$. The concentrations of QC samples were $2.5,250$, and $750 \mathrm{ng} / \mathrm{mL}$, respectively. All working solutions were stored at $4^{\circ} \mathrm{C}$.

\section{Plasma Sample Processing}

$50 \mu \mathrm{L}$ of rat plasma was placed in an Eppendorf (EP) tube, and $10 \mu \mathrm{L}$ of IS $(200 \mathrm{ng} / \mathrm{mL})$ and $150 \mu \mathrm{L}$ acetonitrile were added for protein precipitation. The mixture was vortexed for 1.0 minute, and then 10 minutes at $10,000 \mathrm{rmp}$ was centrifuged. $50 \mu \mathrm{L}$ of the supernatant was moved and detected, and the injection volume was $2 \mu \mathrm{L}$.

\section{Method Validation}

According to the main regulatory principles of the US FDA, we have verified the integral validation of the bioanalytical approach, including: calibration curve, selectivity, LLOQ, accuracy, precision, matrix effect, recovery, and stability. ${ }^{17}$

\section{Statistical Analysis}

In this experiment, the pharmacokinetic parameters of Selinexor were calculated using DAS 2.0 software. SPSS 18.0 software was used for data processing, and the main pharmacokinetic parameters between different groups were compared. $\mathrm{P}<0.05$ was considered to be of statistical significance.

\section{Results and Discussion}

\section{Method Development and Optimization}

The UPLC-MS/MS has been widely used in the analysis and determination of samples in various biological matrices because of its faster analysis time and high sensitivity. As we

Table 2 The MS Parameters Parameters of Selinexor and IS

\begin{tabular}{|l|c|c|c|c|c|c|}
\hline Analytes & ESI Source & RT (min) & Parent (m/z) & Daughter (m/z) & Cone (V) & Collision (V) \\
\hline Selinexor & + & 1.76 & 443.95 & 333.90 & 30 & 15 \\
IS & + & 1.59 & 525.12 & 98.300 & 30 & 20 \\
\hline
\end{tabular}


all know, the choice of column was crucial. We evaluated the retention time of the analyte and the state of the peak shape, and finally selected the Acquity BEH C18 column $(2.1 \mathrm{~mm} \times$ $50 \mathrm{~mm}, 1.7 \mu \mathrm{m})$. The composition of the mobile phase affects sensitivity and peak shape, as well as the retention time and background noise of the analyte. We evaluated different combinations of acetonitrile, methanol, and acetic acid, respectively. Through verification, it was found that formic acid can enhance the chromatographic signal of the analyte and IS. The gradient elution mobile phase can achieve better peak-out effect. The temperature of the mobile phase was $45^{\circ} \mathrm{C}$.

In this study, higher response values were obtained by improving the conditions set by the mass spectrometer. Mass spectrometry analysis was performed on the positive ionization interface of electrospray ionization (ESI) using XEVO TQ-S triple quadrupole mass spectrometer to optimize the main parameters, such as air pressure, fragmentation energy and ion spray voltage. The internal standard is fedratinib because it is stable and easy to obtain, and it has good separation from selinexor, good peak shape, and similar retention time. Endogenous substances in rat plasma samples do not affect the determination of selinexor and IS concentrations.

After screening, plasma samples were processed using acetonitrile. This method was suitable for processing a large number of samples in DDIs studies. Through gradient elution, the interference of endogenous substances was effectively removed. In addition, the acetonitrile protein precipitation method could meet the requirements of high-throughput testing.

\section{Specificity}

Observe the interference of endogenous substances and chromatographic integrity by comparing the blank plasma samples, the blank plasma samples containing the target analytes of selinexor and IS, and the samples from oral selinexor $3 \mathrm{~h}$ in rats were processed. The results showed that the method has good selectivity, no interference peaks of endogenous substances were observed, the peak out times of analyte and IS peaks were 1.76 and $1.59 \mathrm{~min}$, respectively (Figure 2).

\section{Linearity and Carryover}

The standards were added to the blank plasma of rats, and samples with concentrations of 1, 5, 10, 50, 100, 200, 500, $1000 \mathrm{ng} / \mathrm{mL}$ were prepared, the calibration curve was evaluated at three consecutive days by UPLC-MS/MS. The calibration curve was plotted against the theoretical concentration (x) of each analyte and using the weighting of the peak area ratio of selinexor to IS $\left(1 / \mathrm{x}^{2}\right)$. The standard curve of selinexor was: $\mathrm{y}=$ $0.6674 * x+1.1795\left(r^{2}=0.9962\right)$. The lower limit of quantitation was $1 \mathrm{ng} / \mathrm{mL}$. The carryover test was based on the fact that

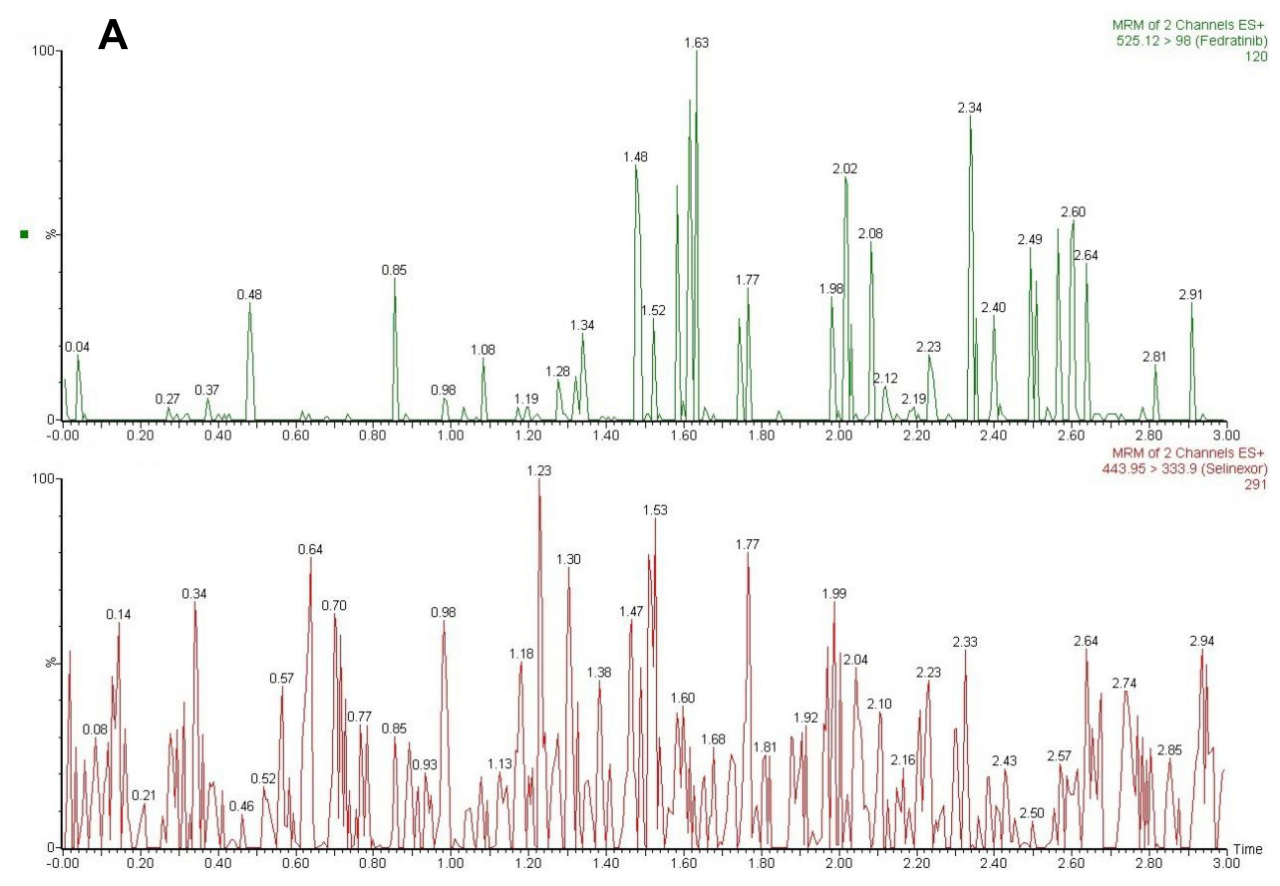

Figure 2 Contiued. 

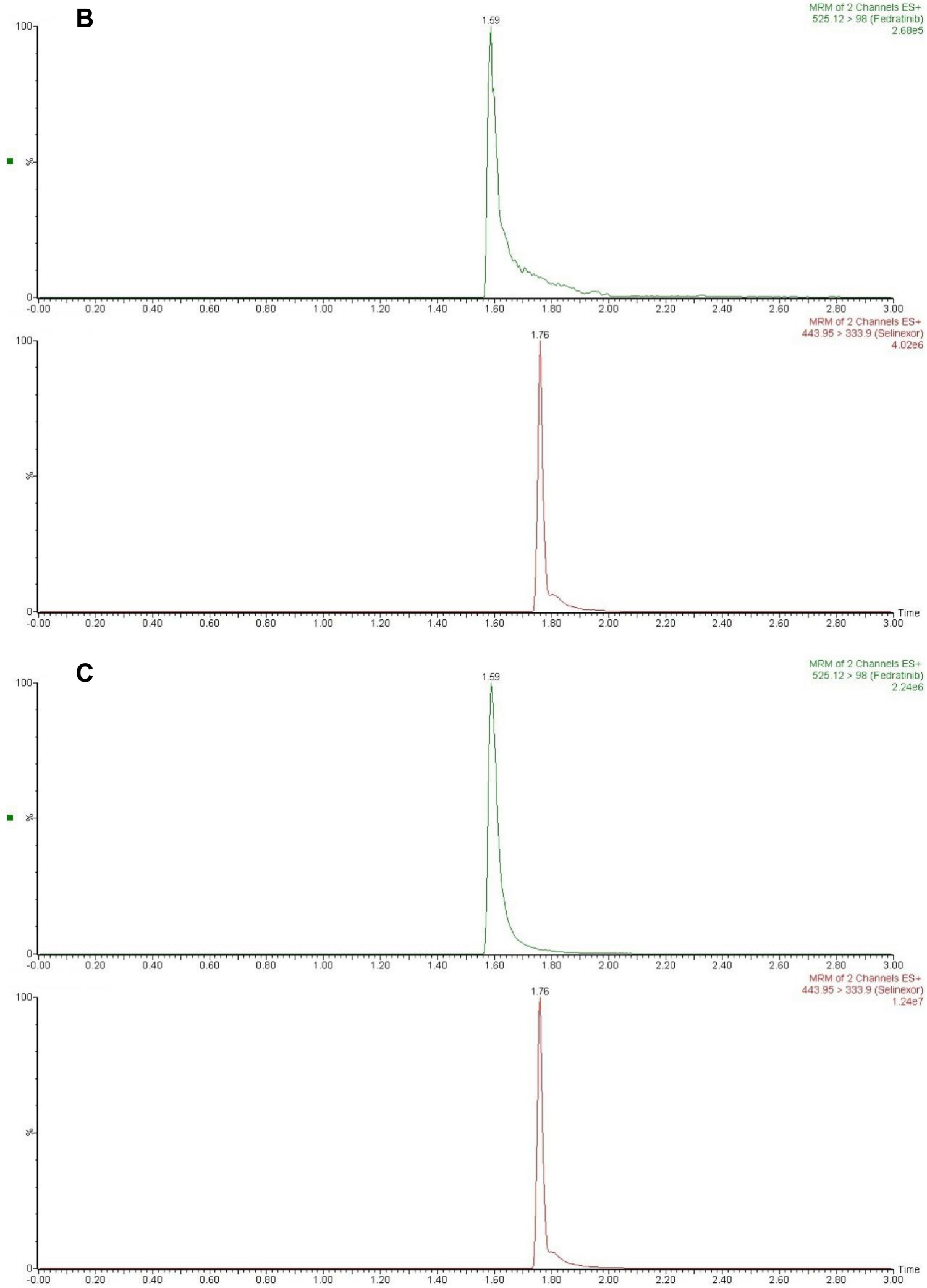

Figure 2 Representative chromatograms in positive ion mode. (A) A blank plasma sample; (B) a blank plasma sample spiked with selinexor and IS; (C) a rat plasma sample $3.0 \mathrm{~h}$ after oral administration of selinexor. 
after the sample concentration was $1000 \mathrm{ng} / \mathrm{mL}$, the analyzer does not detect any residual analyte the next time a blank plasma sample was injected. The experimental results showed that the method has a good linear relationship and the carryover does not affect the determination of selinexor.

\section{Precision, Accuracy, Recovery and Matrix Effect (ME)}

Three different concentration levels of 6 QC samples were used to evaluate the inter-day precision (RSD\%) and accuracy $(\mathrm{RE} \%)$ at three consecutive days, while the intra-day accuracy and precision were calculated in a day. The results are shown in Table 3 . The recovery rate was expressed by the ratio of the chromatographic peak area of selinexor in the QC sample repeated six times to the chromatographic peak area of the analyte in the blank plasma sample processed at the corresponding concentration level. The ME was evaluated by comparing the peak area ratio of selinexor incorporated into the extracted blank matrix to the pure reference standard solution. The recovery and ME results are shown in Table 4 . The results show that the precision, accuracy, recovery and ME of selinexor met the relevant regulations. In this experiment, the accuracy and precision of selinexor was good, the recovery rate of the experimental method was high.

\section{Stability}

Under the three concentration conditions, and 6 replicates were obtained for each concentration, and the stability of QC samples under different treatment conditions was studied (Table 4). The QC samples were placed at room temperature for 4 hours, $4^{\circ} \mathrm{C}$ for 24 hours, 3 freeze-thaw cycles $\left(-20 \sim 25^{\circ} \mathrm{C}\right)$, and $-20^{\circ} \mathrm{C}$ for 4 weeks. The results are shown in Table 5, and passed the required accuracy and precision and met the requirements.

\section{Pharmacokinetic DDIs Study}

The newly established UPLC-MS/MS method was used to detect selinexor in rat plasma and observe the interaction between posaconazole and selinexor. The relationship between the mean concentration levels of selinexor in rat plasma and the time in group A ( $8 \mathrm{mg} / \mathrm{kg}$ selinexor), and group B (20 mg/kg posaconazole and $8 \mathrm{mg} / \mathrm{kg}$ selinexor) are shown in Figure 3. The main pharmacokinetic parameters of selinexor are also shown in Table 6.

Table 3 Precision and Accuracy of Selinexor in Rat Plasma $(n=6$, Mean \pm SD)

\begin{tabular}{|l|c|c|c|c|}
\hline \multirow{2}{*}{ Spiked (ng/mL) } & \multicolumn{2}{|c|}{ Intra-Day } & \multicolumn{2}{|c|}{ Inter-Day } \\
\cline { 2 - 5 } & RSD (\%) & RE (\%) & RSD (\%) & RE (\%) \\
\hline 2.5 & 1.54 & -0.26 & 5.66 & -1.03 \\
250 & 3.95 & 1.29 & 4.73 & -1.52 \\
750 & 2.57 & 0.60 & 2.12 & 2.20 \\
\hline
\end{tabular}

Table 4 The Recoveries and ME of Selinexor and IS in Rat Plasma $(n=6$, Mean \pm SD)

\begin{tabular}{|l|c|c|c|}
\hline Compounds & Spiked $(\mathbf{n g} / \mathbf{m L})$ & Recoveries (\%) & ME (\%) \\
\hline Selinexor & 2.5 & $86.41 \pm 4.98$ & $97.12 \pm 5.59$ \\
& 250 & $88.67 \pm 5.92$ & $101.05 \pm 5.14$ \\
& 750 & $89.59 \pm 4.06$ & $100.79 \pm 2.21$ \\
\hline IS & 200 & $83.66 \pm 2.78$ & $101.98 \pm 9.13$ \\
\hline
\end{tabular}

Table 5 The Stability of Selinexor and IS in Rat Plasma $(n=6$, Mean \pm SD)

\begin{tabular}{|l|c|c|c|c|c|c|c|c|}
\hline \multirow{2}{*}{ Spiked (ng/mL) } & \multicolumn{2}{|c|}{ Room Temperature, 4 h } & \multicolumn{2}{|c|}{ Autosampler 4 ${ }^{\circ} \mathbf{C}, \mathbf{2 4} \mathbf{~ h}$} & \multicolumn{2}{|c|}{ Three Freeze-Thaw } & \multicolumn{2}{c|}{$-20^{\circ} \mathbf{C}, \mathbf{4}$ Weeks } \\
\cline { 2 - 9 } & RSD (\%) & RE (\%) & RSD (\%) & RE (\%) & RSD (\%) & RE (\%) & RSD (\%) & RE (\%) \\
\hline 2.5 & 3.68 & 1.52 & 4.94 & 5.42 & 4.60 & -3.61 & 5.35 & 4.49 \\
250 & 4.98 & -2.64 & 4.76 & 3.46 & 7.86 & 3.44 & 6.97 & -2.62 \\
750 & 2.03 & 2.73 & 2.81 & 1.37 & 2.21 & 1.62 & 2.02 & -1.74 \\
\hline
\end{tabular}




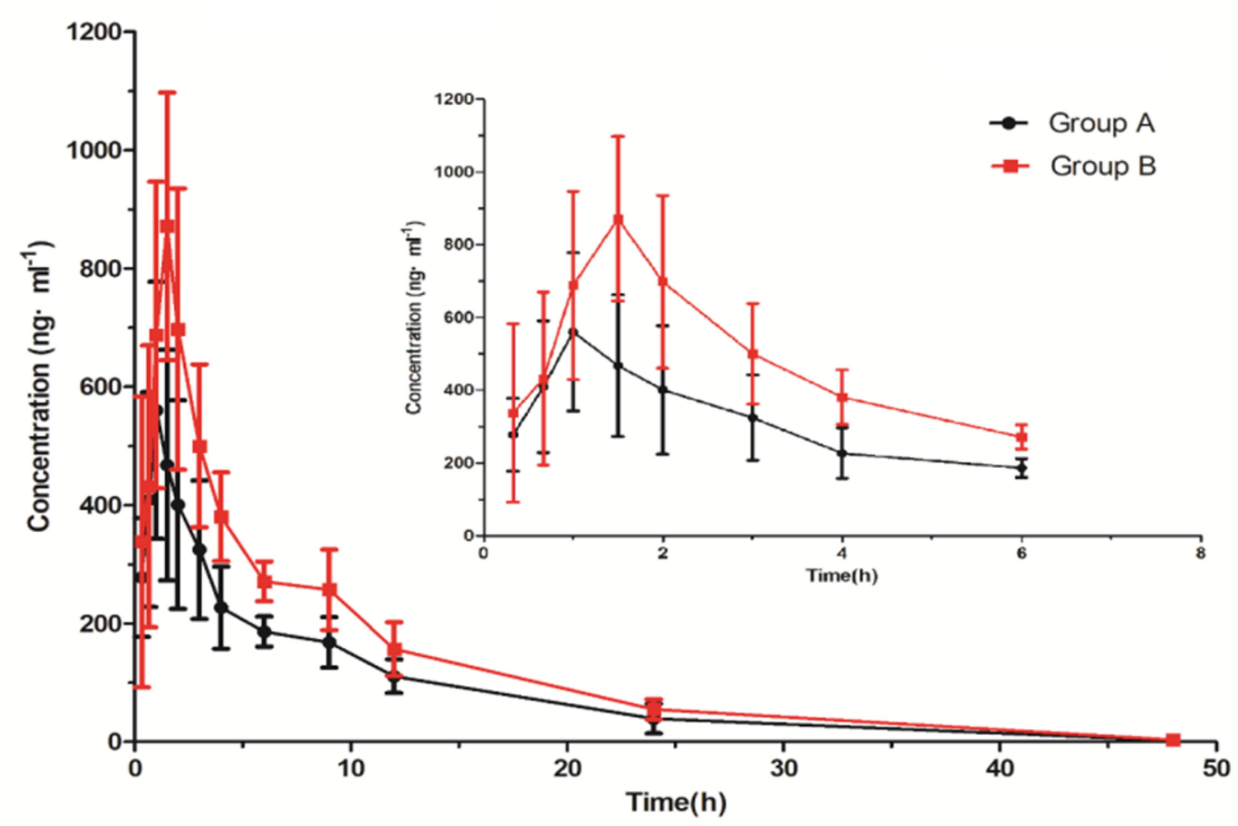

Figure 3 The mean plasma concentration-time curve of selinexor in group A and B after oral administration $8 \mathrm{mg} / \mathrm{kg}$ selinexor (zoomed I to $6 \mathrm{~h}$ pharmacokinetic profile).

The experimental results showed that after the rats were given posaconazole and selinexor at the same time, the $C_{\max }$, $\mathrm{AUC}_{(0-\mathrm{t})}$ and $\mathrm{AUC}_{(0-\infty)}$ of selinexor in group $\mathrm{B}$ were $60.33 \%, 48.28 \%$ and $48.27 \%$ higher than group A, respectively. The $\mathrm{T}_{\max }$ of the experimental group were also extended, and $\mathrm{CLz} / \mathrm{F}$ were also significantly reduced. This shows that posaconazole can increase the exposure of selinexor in the plasma of rats, and the pharmacokinetic process of selinexor was significantly affected by posaconazole.

Previous research results show that isavuconazole, itraconazole, and fluconazole have significant inhibitory effects on selinexor pharmacokinetics and increased selinexor plasma exposure in rats. ${ }^{18}$ Due to certain differences in metabolism in different species, the DDIs between posaconazole and selinexor should be further studied. We will continue to investigate the effect of other CYP3A4 inhibitors (for example, some anti-tumor Chinese medicine) on selinexor in subsequent experiments.

\section{Conclusion}

In summary, this experiment established a new, simple, fast, and accurate UPLC-MS/MS method, which can be applied to the detection of selinexor in rat plasma. In addition, we found that posaconazole can increase selinexor exposure and inhibit its metabolism in rats. Therefore, when selinexor and

Table 6 Pharmacokinetic Parameters of Selinexor After Oral Administration of $8 \mathrm{mg} / \mathrm{kg}$ Selinexor in Group A and B ( $\mathrm{n}=6$, Mean \pm SD)

\begin{tabular}{|l|c|c|}
\hline Parameters & Group A & Group B \\
\hline $\mathrm{t}_{1 / 2}(\mathrm{~h})$ & $6.40 \pm 1.06$ & $6.62 \pm 0.59$ \\
$\mathrm{~T}_{\max }(\mathrm{h})$ & $1.08 \pm 0.20$ & $1.67 \pm 0.26^{* *}$ \\
$\mathrm{MRT}_{(0-\mathrm{t})}(\mathrm{h})$ & $9.39 \pm 2.34$ & $9.10 \pm 1.06$ \\
$\mathrm{MRT}_{(0-\infty)}(\mathrm{h})$ & $9.69 \pm 2.40$ & $9.37 \pm 1.04$ \\
$\mathrm{Cmax}(\mathrm{ng} / \mathrm{mL})$ & $565.86 \pm 207.41$ & $907.23 \pm 218.74^{* *}$ \\
$\mathrm{CLz} / \mathrm{F}(\mathrm{L} / \mathrm{h} / \mathrm{kg})$ & $1.94 \pm 0.35$ & $1.32 \pm 0.26^{* *}$ \\
$\mathrm{AUC}$ & $(0-\mathrm{t})$ \\
$\mathrm{AUC}_{(0-\infty)}(\mathrm{ng} \cdot \mathrm{h} / \mathrm{mL})$ & $4202.91 \pm 692.40$ & $6231.99 \pm 1163.22^{*}$ \\
\hline
\end{tabular}

Note: Compared with the group $\mathrm{A}, * \mathrm{P}<0.05 ; * * \mathrm{P}<0.01$.

Abbreviations: $t_{1 / 2}$, half-life; $T_{\max }$, time of peak concentration; $M R T_{(0-t)}$, mean residence time of 0-t time; $M_{R T} T_{(0-\infty)}$, mean residence time of 0-infinity time; $C_{\max }$, peak concentration; $\mathrm{AUC}_{(0-\mathrm{t})}$, area under curve of 0 -t time; $\mathrm{AUC}_{(0-\infty)}$, area under curve of 0 -infinity time. 
posaconazole were used in combination, the dosage should be adjusted to avoid adverse reactions in clinic.

\section{Data Sharing Statement}

The data used to support the findings of this study are available from the corresponding authors upon request. The corresponding authors: Xiang-jun QIU (lyxiangjun@126.com)

\section{Author Contributions}

All authors made a significant contribution to the work reported, whether that is in the conception, study design, execution, acquisition of data, analysis and interpretation, or in all these areas; took part in drafting, revising or critically reviewing the article; gave final approval of the version to be published; have agreed on the journal to which the article has been submitted; and agree to be accountable for all aspects of the work.

\section{Disclosure}

The authors have declared that no competing interests exist.

\section{References}

1. Karyopharm Therapeutics Inc. XPOVIO ${ }^{\mathrm{TM}}$ (selinexor) tablets, for oral use: US prescribing information; 2019. Available from: www.fda.gov. Accessed August 2, 2019.

2. Gounder MM, Zer A, Tap WD, et al. Phase IB study of selinexor, a first-in-class inhibitor of nuclear export, in patients with advanced refractory bone or soft tissue sarcoma. J Clin Oncol. 2016;34 (26):3166-3174. doi:10.1200/JCO.2016.67.6346.

3. Abdul Razak AR, Mau-Soerensen M, Gabrail NY, et al. First-in-class, first-in-human Phase I study of selinexor, a selective inhibitor of nuclear export, in patients with advanced solid tumors. $J$ Clin Oncol. 2016;34(34):4142-4150. doi:10.1200/JCO.2015.65.3949.

4. Gandhi UH, Senapedis W, Baloglu E, et al. Clinical implications of targeting XPO1-mediated nuclear export in multiple myeloma. Clin Lymphoma Myeloma Leuk. 2018;18(5):335-345. doi:10.1016/j. clml.2018.03.003.

5. Turner JG, Sullivan DM. CRM1-mediated nuclear export of proteins and drug resistance in cancer. Curr Med Chem. 2008;15:2648-2655. doi: $10.2174 / 092986708786242859$

6. Garg M, Kanojia D, Mayakonda A, et al. Selinexor (KPT-330) has antitumor activity against anaplastic thyroid carcinoma in vitro and in vivo and enhances sensitivity to doxorubicin. Sci Rep. 2017;7(1):9749. doi:10.1038/s41598-017-10325-x.

Drug Design, Development and Therapy

\section{Publish your work in this journal}

Drug Design, Development and Therapy is an international, peerreviewed open-access journal that spans the spectrum of drug design and development through to clinical applications. Clinical outcomes, patient safety, and programs for the development and effective, safe, and sustained use of medicines are a feature of the journal, which has also
7. Ranganathan P, Kashyap T, Yu X, Meng X, Garzon R. XPO1 inhibition using selinexor synergizes with chemotherapy in acute myeloid leukemia (AML) by targeting DNA repair and restoring topoisomerase II $\alpha$ to the nucleus. Clin Cancer Res. 2016;22(24):6142. doi:10.1158/1078-0432.CCR-15-2885.

8. Liu XJ, Chong YL, Tu YM, et al. CRM1/XPO1 is associated with clinical outcome in glioma and represents a therapeutic target by perturbing multiple core pathways. J Hematol Oncol. 2016;9:108. doi:10.1186/s13045-016-0338-2.

9. Saulino DM, Younes PS, Biley JM, Younes M. Expression of exportin 1 (XPO1/CRM1) in pancreatic adenocarcinoma. $J$ Clin Oncol. 2017;35(4suppl):327. doi:10.1200/JCO.2017.35.4_suppl.327.

10. Corno C, Stucchi S, De Cesare M, et al. FoxO-1 contributes to the efficacy of the combination of the XPO1 inhibitor selinexor and cisplatin in ovarian carcinoma preclinical models. Biochem Pharmacol. 2018;147:93-103. doi:10.1016/j.bcp.2017.11.009.

11. Nair JS, Musi E, Schwartz GK. Selinexor (KPT-330) induces tumor suppression through nuclear sequestration of IkappaB and downregulation of survivin. Clin Cancer Res. 2017;23(15):4301-4311. doi:10.1158/1078-0432.CCR-16-2632.

12. Karyopharm Therapeutics. Karyopharm announces FDA approval of XPOVIO $^{\text {TM }}$ (selinexor) for the treatment of patients with relapsed or refractory multiple myeloma [media release]. 2019.

13. Karyopharm Receives Positive CHMP Opinion for NEXPOVIO ${ }^{\circledR}$ (selinexor) for the Treatment of Patients with Refractory Multiple Myeloma. Available from: https://investors.karyopharm.com/202101-29-Karyopharm-Receives-Positive-CHMP-Opinion-forNEXPOVIO-R-selinexor-for-the-Treatment-of-Patients-withRefractory-Multiple-Myeloma. Accessed March 26, 2021.

14. Lin Q, Xie S, Qiu X, Chen J, Xu RA. Drug-drug interaction study of imatinib and voriconazole in vitro and in vivo. Infect Drug Resist. 2019;12:1021-1027. doi:10.2147/IDR.S199526

15. Li SL, Zhu YL, Zhu CY, et al. Simultaneous determination of parecoxib and its metabolite valdecoxib concentrations in beagle plasma by UPLC-MS/MS and application for pharmacokinetics study. Drug Des Devel Ther. 2020;14:1117-1125. doi:10.2147/DDDT.S226349

16. Zhou W, Li SL, Zhao T, et al. Effects of dexmedetomidine on the pharmacokinetics of dezocine, midazolam and its metabolite 1-hydroxymidazolam in beagles by UPLC-MS/MS. Drug Des Devel Ther. 2020;14:2595-2605. doi:10.2147/DDDT.S254055

17. US Food and Drug Administration. Guidance for industry: bioanalytical method validation. Rockville, MD, USA: US Department of Health and Human Services, US FDA, Center for Drug Evaluation and Research; 2018. Available from: https://www.fda.gov/regulatoryinformation/search-fda-guidance-documents/bioanalyticalmethodvalidation-guidance-industry. Accessed August 10, 2018.

18. Li SL, Zhang Y, Cheng QS, et al. UPLC-MS/MS measurement of the effect of isavuconazole, itraconazole and fluconazole on the pharmacokinetics of selinexor in rats. Infect Drug Resist. 2020;13:31533161. doi:10.2147/IDR.S269831 and fair peer-review system, which is all easy to use. Visit http://www. dovepress.com/testimonials.php to read real quotes from published authors.

\section{Dovepress}

